\title{
Granulomatosis With Polyangiitis Masquerading as Bilateral Trigeminal Neuralgia
}

\author{
Vivek Joseph ${ }^{\text {a, d }}$, Rahul Anil ${ }^{\text {b, d, e }}$, Neil Gonter ${ }^{c, d}$
}

\begin{abstract}
A 32-year-old woman presented with symptoms of bilateral trigeminal neuralgia $(\mathrm{TN})$ that has been refractory to empiric therapy. Imaging showed normal caliber trigeminal nerves with no evidence of nerve compression. Autoimmune etiology was considered due to unnatural presentation of symptoms. Workup revealed elevated perinuclear antineutrophil cytoplasmic antibody (P-ANCA) and elevated myeloperoxidase (MPO) antibodies suggestive of localized ANCArelated vasculitis. A literature review revealed one case, which illustrates granulomatosis with polyangiitis (GPA) causing the classical symptoms of TN in absence of its more common symptoms. Patient was started on methotrexate $15 \mathrm{mg}$ weekly and low-dose prednisone daily with significant improvement in symptoms. Ear-nose-throat (ENT) manifestations of rheumatologic disorders represent a diagnostic challenge. ENT symptoms can often represent the initial sign of an otherwise asymptomatic and undiagnosed autoimmune disease process, which often calls for prompt and aggressive immunosuppressive treatment which leads to not only better control of TN symptoms but also we speculate that early immunosuppressive therapy will control the progression of the underlying autoimmune disease.
\end{abstract}

Keywords: Rheumatology; Mixed connective tissue disorders; Vasculitis; Trigeminal neuralgia; Immunosuppressive therapy

\section{Introduction}

Granulomatosis with polyangiitis (GPA) is one of the antineu-

\footnotetext{
Manuscript accepted for publication August 08, 2016

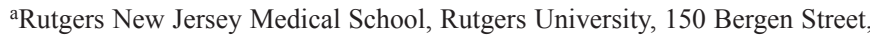
Newark, NJ 07601, USA

${ }^{b}$ Department of Neurology, Yale School of Medicine, Yale University, 15 York Street, New Haven, CT 06510, USA

'Hackensack University Medical Centre, 30 Prospect Avenue, Hackensack, NJ 07601, USA

${ }^{\mathrm{d} A l l}$ authors contributed equally.

${ }^{\text {e}}$ Corresponding Author: Rahul Anil, Department of Neurology, Yale School of Medicine, Yale University, 15 York Street, New Haven, CT 06510, USA. Email: drrahulanil@gmail.com
}

doi: http://dx.doi.org/10.14740/jmc2636e trophil cytoplasmic antibody (ANCA)-associated vasculitides with a predilection to affect the respiratory tract and kidneys. Ear-nose-throat (ENT) manifestations of GPA such as bony and cartilage destruction resulting in saddle nose deformity, nasal crusting, sinusitis, otitis media, persistent rhinorrhea, purulent/bloody nasal discharge, oral and/or nasal ulcers, and polychondritis are commonly reported in literature [1]. We present a rare presentation of GPA with symptoms of isolated bilateral trigeminal neuralgia (TN).

\section{Case Report}

A 32-year-old Caucasian female with past medical history of asthma and migraine headaches presented with complaints of paroxysms of severe lancinating-pain down the jaws. Symptoms were usually unilateral but occasionally simultaneous on both sides. Chewing and yawning precipitated pain and worsened in cold weather. She also had diffuse pain in both shoulders and Raynaud's symptoms in the left hand. Physical examination revealed no neurological deficits. She was diagnosed to have TN and was started on carbamazepine $200 \mathrm{mg}$ twice daily and nortriptyline with no significant improvement in symptoms. Due to lack of improvement, MRI of brain was done, which showed normal caliber trigeminal nerves bilaterally with no abnormal enhancement with contrast. Therefore, autoimmune etiology was considered in view of bilateral nature of symptoms and lack of relief from initial empiric therapy. ANA screen was abnormal but RF, anti-DS DNA, antiRNP, anti-SM, anti-SSA and SSB were all negative. Further workup revealed elevated P-ANCA and elevated myeloperoxidase (MPO) antibodies suggestive of localized ANCA-related vasculitis. Serum creatinine, GFR and urine analysis were within normal limits. She was started on methotrexate $15 \mathrm{mg}$ weekly and low-dose prednisone. The option of biopsy to confirm diagnosis was presented to the patient. However, patient declined citing the significant improvement in symptoms by above mentioned treatment.

\section{Discussion}

$\mathrm{TN}$ is one of the most common causes of orofacial pain. It is defined clinically by the presence of paroxysmal, stereotyped attacks of usually intense, sharp, superficial, or stabbing pain in the distribution of one or more branches of the trigeminal 
nerve [2]. Severe symptoms are often associated with facial muscle spasms; this finding gave rise to the classical French term for this disorder, Tic Douloureux. The most common etiology (accounting for $80-90 \%$ of cases) for $\mathrm{TN}$ is compression of the trigeminal nerve by an aberrant loop of artery or vein.

Other causes of TN from nerve compression include vestibular schwannoma, meningioma, epidermoid or other cyst, or, rarely, a saccular aneurysm or arteriovenous malformation [3]. All of these usually cause unilateral symptoms. Bilateral and especially simultaneous bilateral symptoms should arouse the suspicion of a systemic cause.

In this case report, we present a patient with recent onset paroxysms of jaw pain. Symptoms were classical for TN, with no neurological deficits elicitable clinically. MRI of the brain with contrast showed no evidence of compression of the trigeminal nerves. The bilateral and especially simultaneous nature of symptoms made us suspect a systemic cause. History of occasional joint pains and Raynaud's phenomena of the hands raised the suspicion that the TN was a manifestation of an otherwise occult rheumatologic process. Further workup revealed elevated P-ANCA and MPO antibodies, suggestive of localized GPA. A review of literature reveals that commonly rheumatologic causes for $\mathrm{TN}$ are systemic lupus erythematosus, systemic sclerosis and mixed connective tissue disease [4]. To our knowledge, there has only been one prior case reported in literature that describes GPA causing the classical symptoms of TN with no evidence of skin or submucosal ulcerations [5].

ENT manifestations of rheumatologic disorders represent a diagnostic challenge. ENT symptoms can often represent the initial sign of an otherwise asymptomatic and undiagnosed autoimmune disease process, which often calls for prompt and aggressive immunosuppressive treatment [6]. Moreover, in patients with established autoimmune disease, ENT symptoms such as B/L TN may be often being overlooked by both the physicians who are usually preoccupied with the main manifestations of the disease. Hence we recommend that in all patients with bilateral or poorly controlled symptoms of TN with no evidence of nerve compression on imaging, a concerted effort must be made to look for a systemic cause for the symptoms. Not only will treatment of the systemic autoimmune etiology lead to better control of TN symptoms but also we speculate that early immunosuppressive therapy will control the progression of the underlying autoimmune disease.

\section{Conclusion}

In conclusion, we address the following. ENT manifestations of otherwise silent autoimmune disease represent a significant diagnostic challenge. Systemic etiology must be looked for in patients with bilateral/poorly controlled TN. Early immunosuppressive therapy will likely lead to better control of the progression of the underlying dormant autoimmune disease.

\section{Conflicts of Interest}

All authors declare no conflicts of interest.

\section{References}

1. Watts R, Lane S, Hanslik T, Hauser T, Hellmich B, Koldingsnes W, Mahr A, et al. Development and validation of a consensus methodology for the classification of the ANCA-associated vasculitides and polyarteritis nodosa for epidemiological studies. Ann Rheum Dis. 2007;66(2):222-227.

2. The International Classification of Headache Disorders, 3rd edition (beta version). Cephalalgia. 2013;33(9):629808.

3. Love S, Coakham HB. Trigeminal neuralgia: pathology and pathogenesis. Brain. 2001;124(Pt 12):2347-2360.

4. Papadimitraki ED, Kyrmizakis DE, Kritikos I, Boumpas DT. Ear-nose-throat manifestations of autoimmune rheumatic diseases. Clin Exp Rheumatol. 2004;22(4):485494.

5. Shah Parvaiz A, Iffat Hassan, Tariq Qureshi, Rauf Ahmad, Rakesh K Koul. Wegener's Granulomatosis Masquerading as Trigeminal Neuralgia. JIACM. 2010;11(2):153154.

6. Alfaro-Giner A, Penarrocha-Diago M, Bagan-Sebastian JV. Orofacial manifestations of mixed connective tissue disease with an uncommon serologic evolution. Oral Surg Oral Med Oral Pathol. 1992;73(4):441-444. 